\title{
Análisis bioético del impacto de las Técnicas de Reproducción Asistida (TRA) en la salud de niños y madres
}

\section{Bioethical analysis of the impact of Assisted Reproduction Techniques (ART) on the health of children and mothers}

\author{
Ana Herrero García, * Javier Lluna González, ** Justo Aznar Lucea***
}

https://doi.org/10.36105/mye.2020v31n2.02

\section{Resumen}

Las técnicas de reproducción asistida (TRA) pueden estar asociadas a complicaciones obstétricas y perinatales, incluido un mayor riesgo de malformaciones congénitas y de patología adquirida. Es un deber de los profesionales sanitarios informar a quienes desean someterse a estas técnicas, sobre los riesgos para la salud de los hijos y las madres. A parte de los riesgos biológicos comentados, las TRA plantean también múltiples problemas bioéticos, que se han de abordar partiendo del valor fundamental de la vida humana.

\footnotetext{
* Médico pediatra. Servicio de Pediatría Hospital de Llíria. Valencia. España. Correo electrónico: herrero.garcia.ana@gmail.com https://orcid.org/0000-0002-8686-2976

** Doctor en Medicina. Profesor de la Facultad de Medicina y Ciencias de la Salud y Miembro del Observatorio de Bioética. Universidad Católica de Valencia. España. Correo electrónico: jllunag@yahoo.es https://orcid.org/0000-0002-2025-8565

*** Doctor en Medicina. Director del Instituto de Ciencias de la Vida. Universidad Católica de Valencia. España. Correo electrónico: justo.aznar@ucv.es https://orcid.org/0000-0002-6899-4932

Recepción: 22 de noviembre de 2019. Aceptación: 12 de enero de 2020.
} 
El objetivo principal de este estudio retrospectivo es el análisis bioético y biomédico del impacto de las TRA en la salud de los niños y sus madres, comparando el riesgo de patología entre los nacidos por TRA y por concepción natural.

Palabras clave: fertilización in vitro, inyección intracitoplasmática de espermatozoides, efectos adversos, malformaciones congénitas, valoración bioética.

\section{Introducción}

No cabe ninguna duda de que conseguir un hijo para una pareja que tiene dificultades para ello es un bien objetivo. Para conseguirlo, se pueden utilizar las técnicas de reproducción asistida (TRA) o los métodos naturales de regulación de la fertilidad humana.

Las TRA tienen una amplia aceptación social, pues desde que nació la primera niña, Louise Brown, en 1978, por fecundación in vitro, en el mundo nacen más de 200.000 niños al año por estas técnicas; ${ }^{1}$ es decir, más del 3\% de los niños nacidos, ${ }^{2}$ siendo el total de ellos más de 8 millones. ${ }^{3}$

Sin embargo, como cualquier intervención biomédica, las técnicas de reproducción humana asistida no están libres de riesgos. En efecto, al inicio de los años 90 comenzó la alerta pediátrica por los defectos y anomalías que presentaban estos niños, al parecer en proporción mayor que los nacidos de forma natural, ${ }^{4}$ pues numerosos estudios de cohortes retrospectivos demostraban un riesgo aumentado de defectos congénitos, ${ }^{5-7}$ en particular de malformaciones cardiacas y cromosomopatías, asociadas con la fecundación in vitro, ${ }^{8-9}$ aunque otros, en cambio, no detectaban diferencias estadísticamente significativas al estratificarlos según edad materna, paridad y edad gestacional. ${ }^{10}$ Además, en un estudio reciente que analiza el estado de salud en el largo plazo de niños nacidos por 
TRA, no se encuentran diferencias significativas con los nacidos por vía natural. ${ }^{11}$

Cuando se valora si existen diferencias en cuanto al riesgo de malformaciones congénitas según la técnica empleada, fecundación in vitro (FIV) o inyección intracitoplasmática de espermatozoides (ICSI), diversos estudios no las encuentran, ${ }^{12-14}$ aunque otro sí que evidencia un incremento de defectos congénitos tras ICSI, pero no tras FIV. ${ }^{15}$

Respecto de la inseminación intrauterina, también se ha demostrado que conlleva tantos riesgos de anomalías congénitas en la descendencia como la FIV o la ICSI. ${ }^{16}$

En cuanto a la posibilidad de que sufran patologías adquiridas, algunos estudios detectan que los niños nacidos por FIV tienen mayor riesgo de sufrir alteraciones del desarrollo psicomotor, parálisis cerebral, autismo, ${ }^{17,18}$ asma $^{19} \mathrm{y}$, en general, peor estado de salud en la primera infancia. ${ }^{20} \mathrm{~A}$ más largo plazo, se detecta un envejecimiento vascular prematuro, con mayor riesgo de hipertensión arterial. $^{21}$

Otro aspecto que genera preocupación es la fertilidad futura de las personas concebidas por FIV, ya que se ha reportado una peor calidad y cantidad del semen en adultos jóvenes nacidos tras ICSI, posiblemente relacionada con la infertilidad del padre. ${ }^{22}$

En cuanto a la causa de los problemas que padecen los niños nacidos por TRA, parece que se debe a una combinación de la subfertilidad parental y a las técnicas en sí mismas, sin perder de vista el mayor número de embarazos múltiples que se dan en las TRA, ${ }^{23}$ y que, como se sabe, suelen presentar mayores problemas que los embarazos de un solo feto. ${ }^{24}$

También podrían estar implicadas las mutaciones epigenéticas, ${ }^{25}$ que podrían darse principalmente durante la formación de los gametos, la fecundación y las primeras fases del desarrollo embrionario.

En cuanto a las madres que se someten a las TRA, se ha demostrado que presentan mayor riesgo de patología obstétrica, fundamentalmente por el síndrome de hiperestimulación ovárica. ${ }^{26}$ 
En el presente artículo se estudia el impacto de las TRA en la salud de los niños concebidos por estas técnicas, comparándolos con los nacidos por concepción natural $(\mathrm{CN})$ en el Hospital Universitario La Fe, de Valencia, España, a la vez que se realiza una más amplia evaluación de los problemas bioéticos que pueden acompañar a las TRA.

\section{Material y métodos}

Se trata de un estudio observacional retrospectivo de cohortes de recién nacidos en el año 2016 en el Hospital La Fe de Valencia (España). Mediante un muestreo aleatorio simple, se seleccionaron 300 niños procedentes de concepción natural y 300 procedentes de TRA. En ambos grupos hay aproximadamente el mismo número de recién nacidos de cada sexo, aunque con ligero predominio masculino: 157 varones (52.33\%) y 143 mujeres (47.67\%).

Se consideraron como criterios de exclusión a los nacidos vivos fallecidos posteriormente por cualquier causa, y a aquéllos nacidos vivos sin datos de seguimiento en los sistemas de registro de la historia clínica electrónica del Hospital.

Las variables evaluables son: edad materna, edad gestacional, tipo de embarazo y número de hijos, peso al nacimiento, tipo de parto, exposición a tóxicos de la madre y su tipo, patología gestacional, sufrimiento fetal, antecedentes familiares de enfermedades genéticas, ingreso al nacimiento, malformaciones congénita y su tipo (según la clasificación del CIE-10), patología adquirida hasta los 2 años de edad y su tipo (se incluyen las enfermedades codificadas en el CIE-10, excepto las patologías infecciosas y las enfermedades transitorias producidas totalmente por factores mecánicos externos).

Además, en la cohorte de niños-TRA, se recoge el tipo de técnica (FIV convencional, ICSI e inseminación artificial o IA), motivo de 
las TRA (factor materno, paterno, mixto), y la procedencia de los gametos (propios o de donante).

Los datos se expresan mediante media (desviación estándar), mediana (1 er., 3er. cuartil) en el caso de las variables numéricas, y mediante la frecuencia absoluta porcentual para las variables cualitativas.

Para evaluar las posibles diferencias entre los grupos TRA y CN se ajustan regresiones logísticas multivariables. Como factores de confusión se introducen las variables de edad materna, edad gestacional, tóxicos y multiparidad.

Para contemplar el efecto no lineal de la edad se añaden «splines» de tercer grado en el modelo. Adicionalmente, para valorar la asociación entre edad gestacional y multiparidad se utiliza un modelo de regresión lineal. Asimismo, para relacionar multiparidad con las TRA se ajusta una regresión logística. Todos los análisis se realizan mediante el software estadístico R (versión 3.6.1).

\section{Resultados}

Durante 2016 nacieron 5,292 niños en el Hospital La Fe, de los cuales $4,799(90.6 \%)$ fue por $\mathrm{CN}$ y $493(9.3 \%)$ por TRA, cuyas características se especifican en la Tabla 1 . Los datos referentes a la edad materna por tramos de edades se recogen en la Tabla 2.

En cuanto al porcentaje de cesáreas, fue 3 veces mayor en el grupo de TRA que en el de CN. Por otro lado, en el grupo de TRA el $51 \%$ de los nacidos lo fueron de embarazos múltiples, mientras que en el grupo de $\mathrm{CN}$ dicho porcentaje fue del 3.67\%. Se registró un solo trillizo en el grupo de $\mathrm{CN}$, frente a 5 en el grupo de TRA (Tabla 3).

En cuanto a patología materna, el 17\% de las madres-CN presentaron algún tipo de patología gestacional, frente al 37\% de las madres-TRA. 
Por otro lado, el porcentaje de niños prematuros y sus características se especifican en la Tabla 4. En ella se observa que el 6\% de los niños TRA presentaban bajo peso al nacimiento, frente al $0.66 \%$ de los niños-CN (Tabla 5).

En ambos grupos nacieron 3 niños con pérdida de bienestar fetal al nacimiento. También se aprecia un mayor porcentaje de ingresos al nacimiento en el grupo TRA $(28,3 \%)$, frente a $6,6 \%$ en el grupo CN.

El porcentaje de niños con antecedentes nacidos de padres con enfermedades hereditarias también fue mayor en los niños TRA $(8.33 \%)$ frente a un $1.33 \%$ en $\mathrm{CN}$.

De la cohorte de niños-TRA, el 91\% fue producto de una FIV y el $9 \%$ de inseminación artificial (IA). Aproximadamente la cuarta parte de las FIV se hicieron mediante ICSI, aunque este dato esté probablemente infraestimado por falta de registro en las historias clínicas.

Las TRA se realizaron por causa materna en el 53\% de los casos; por causa paterna en el $11 \%$ y por causa mixta en el $27 \%$.

Aunque en la mayoría de los niños-TRA se utilizaron gametos de sus progenitores, en más del 30\% procedían de donantes.

En cuanto a los problemas médicos, los niños TRA los presentaron en el $56.67 \%$ de los casos y en el $43,33 \%$ en el grupo CN. En el grupo TRA se detectó un 19\% de malformaciones congénitas, frente a un $9.3 \%$ en el grupo $\mathrm{CN}$.

Las patologías más frecuentes en el grupo TRA fueron las malformaciones cardiacas (24 niños), seguidas de las urinarias ( 9 niños), las osteomuscular (7 niños) y las alteraciones cromosómicas (7 niños).

En el caso de los 3 niños-TRA que presentaban retraso psicomotor, se solicitó el estudio genético, el cual confirmó la presencia de mutaciones de novo causantes de la enfermedad. Se dieron dos casos en una gestación gemelar monocorial biamniótica, en la que se planteó una discordancia entre el sexo genético (por la presencia del cromosoma $\mathrm{Y}$ en ambos) y el ecográfico (un gemelo con feno- 
tipo femenino y otro masculino). Hasta el momento no ha tenido repercusión clínica, asignándose el sexo respectivamente según los genitales internos y externos. Los síndromes y cromosomopatías de ambas cohortes se especifican en la Tabla 6.

La frecuencia de patologías adquiridas es mayor en el grupo de niños concebidos por TRA (62\%), que en el grupo de CN (38\%). El tipo de patología más frecuente se describe en el Gráfico 1. No se registró ningún niño con patología oncológica ni reumatológica, probablemente porque la incidencia de esta patología en la infancia suele darse en edades superiores a los dos años.

También se ha podido confirmar que, a menor edad gestacional de los niños, hay mayor riesgo de malformaciones congénitas (OR $=0.913, \mathrm{IC} 95 \%[0.844-0.99], \mathrm{P}=0.023)$, independientemente de la edad de las madres, que hayan tenido exposición a tóxicos, que hayan sido concebidos de forma natural o por técnicas de reproducción asistida. No se puede afirmar que el mayor riesgo de malformaciones congénitas en los niños-TRA sea por la técnica propiamente $(\mathrm{OR}=1.459, \mathrm{IC} 95 \%$ [0.793 - 2.701], $\mathrm{P}=0.225)$.

En cuanto al riesgo de sufrir patología adquirida a lo largo de los 2 primeros años de vida, se observa que está aumentada en niños de menor edad gestacional (OR $=0.743$, IC95\% [0.6690.816], $\mathrm{P}<0.001)$. Además, los niños concebidos por TRA tienen mayor riesgo de presentar patología durante los dos primeros años de vida $(\mathrm{OR}=1.926, \mathrm{IC} 95 \%$ [1.301 - 2.857], $\mathrm{P}=0.001)$ (Tabla 7).

El análisis de la patología gestacional de las madres sometidas a TRA es un objetivo secundario de este estudio, comprobándose que las madres de mayor edad y las que se someten a TRA, presentan mayor riesgo de patología gestacional. A su vez, la presencia de patología durante la gestación también se relaciona de forma estadísticamente significativa con la prematuridad (menor edad gestacional). A mayor edad de las madres, mayor riesgo de patología gestacional $(\mathrm{OR}=5.096, \mathrm{IC} 95 \%$ [1.371-26.44], $\mathrm{P}=0.028)$. A mayor presencia de patología gestacional, menor edad gestacional 
$(\mathrm{OR}=0.918, \mathrm{IC} 95 \%$ [0.856-0.985], $\mathrm{P}=0.018)$. Las madres sometidas a TRA se relacionan con mayor riesgo de patología gestacional $(\mathrm{OR}=1.97, \mathrm{IC} 95 \%$ [1.246 - 3.13], $\mathrm{P}=0.004)$.

También se comprueba que no hay diferente riesgo de presentar patología gestacional $(\mathrm{OR}=0.639, \mathrm{IC} 95 \%[0.279-1.50], \mathrm{P}=$ 0.294), malformaciones congénitas (OR $=1.62$, IC95\% [0.514 7.26], $\mathrm{P}=0.458)$ o patología adquirida (OR $=0.519$, IC95\% [0.191 - 1.27], $\mathrm{P}=0.169)$ en los niños concebidos por FIV respecto de los concebidos por IA, controlando la edad gestacional, la edad materna y los tóxicos de las madres. Tampoco hay diferencias entre las técnicas FIV e ICSI, en cuanto al riesgo de patología gestacional $(\mathrm{OR}=1.548, \mathrm{IC} 95 \%$ [0.877- 2.725], $\mathrm{P}=0.13)$, malformaciones congénitas $(\mathrm{OR}=1.168$, IC95\% $[0.561-2.346], \mathrm{P}=0.669)$ o patología adquirida $(\mathrm{OR}=1.422$, IC95\% [0.794 - 2.593], $\mathrm{P}=$ $0.242)$, controlando las mismas variables.

\section{Ausencia de figura paterna}

Los niños registrados sin figura paterna fueron 29 , casi el $10 \%$ de la cohorte TRA. Dos de estos niños tuvieron 2 madres, y los otros 27 tuvieron madre sin pareja. Cabe destacar que la totalidad de las madres solas y madres lesbianas utilizaron la FIV. El 55\% de estas madres tenía más de 40 años cuando se llevó a cabo el procedimiento. La madre sola de mayor edad registrada tenía 47 años.

Aproximadamente la mitad de los embarazos fueron gemelares. Los embarazos de los niños con dos madres fueron partos únicos, por lo que, si tenemos en cuenta únicamente a las 27 madres sin pareja, 15 de ellas (más de la mitad) afrontaron un embarazo múltiple.

En 16 de las madres, la FIV se llevó a cabo con semen de donante, y en 13 de ellas hubo donación de ambos gametos, siendo su edad avanzada la principal causa de la ovodonación (11 de las 13 eran mayores de 40 años). 
Análisis bioético del impacto de las TRA en la salud de niños y madres

\section{Discusión}

Los datos de este estudio confirman los previamente referidos en la literatura sobre la mayor incidencia de problemas médicos en los niños nacidos por TRA.

Pero en nuestro estudio cabe destacar que los niños nacidos por TRA tienen mayor riesgo de desarrollar patología hasta los 2 años de edad, que los nacidos por $\mathrm{CN}$, independientemente de otros factores que pueden estar directamente relacionados con su aparición (edad materna avanzada, prematuridad, multiparidad, exposición a tóxicos durante la gestación y patología gestacional). Igualmente, estos niños tienen más riesgo por proceder de embarazos múltiples, lo que favorece la prematuridad y esto, a su vez, constituye un riego mayor de padecer anomalías cardiacas, urinarias y osteomusculares. También presentan mayor número de malformaciones congénitas y anomalías cromosómicas.

Las madres sometidas a TRA tienen más riesgo de presentar patología durante la gestación, riesgo que también se ve aumentado por la edad materna más avanzada.

Sin embargo, no se han visto diferencias significativas entre la ICSI, la FIV y la IA.

Por un lado, el hecho de que las malformaciones congénitas estén más frecuentemente asociadas a la prematuridad y, por tanto, más presentes en los niños nacidos mediante TRA, es un dato preocupante, porque las malformaciones congénitas constituyen la primera causa de mortalidad infantil (neonatal y postneonatal) en España. ${ }^{27}$ De hecho, más de la mitad de los niños-TRA fallecidos, que se excluyeron del estudio, presentaban malformaciones congénitas graves, algunas incompatibles con la vida.

Por otro lado, también en España, desde el año 2001 hasta el 2016 las tasas de mortalidad neonatal y postneonatal han descendido un $32.1 \%$ y $35.1 \%$, respectivamente. ${ }^{27}$ Gran parte de este descenso se debe al observado en las defunciones por malformaciones congénitas, deformidades y anomalías cromosómicas, ya 
que representan casi la cuarta parte de todas las defunciones en menores de 1 año. Concretamente, la tasa de mortalidad infantil por esta causa de muerte descendió un 45.1\% entre 2001 y 2016. Si se tiene en cuenta que, con la aprobación de la Ley Orgánica 9/ 1985, se legalizó el aborto en España bajo tres supuestos, ${ }^{28}$ y uno de ellos (el supuesto «eugenésico») permitía abortar niños con malformaciones congénitas, y posteriormente se aprobó la Ley Orgánica 2/2010, vigente actualmente, que legalizó el aborto por malformaciones graves en el feto en cualquier momento del embarazo, ${ }^{29}$ nos parece razonable pensar que ello ha podido influir en la disminución de la mortalidad a causa de malformaciones congénitas.

\section{Valoración ética de las TRA}

Con independencia de los datos médicos anteriormente referidos, las TRA merecen también una reflexión ética.

Es indudable que el que una pareja con problemas de infertilidad pueda conseguir un hijo utilizando las TRA es un bien objetivo, de ahí su gran aceptación social. Sin embargo, es objetivo también que, tanto las madres como los hijos nacidos pueden mostrar efectos médicos adversos, como se ha constatado, tanto en los propios resultados de este trabajo, como en la amplia revisión bibliográfica realizada; pero, además de ello, también es una realidad que en las TRA se pierde un elevado número de embriones humanos. ${ }^{30}$

Todo ello abunda en la necesidad de realizar, aunque sea muy sucintamente, una reflexión bioética sobre estas técnicas.

Además de los efectos adversos ya conocidos que pueden sufrir madres e hijos, y de la pérdida de embriones ya comentada, las TRA presentan otras dificultades bioéticas, como pueden ser: el exceso de embriones que se generan para rentabilizar la técnica, lo que obliga a congelar gran número de ellos, práctica indudablemente no acorde con la dignidad humana de dichos embriones; tampoco existe una solución razonadamente aceptable para ellos, pues la 
mayoría de estos embriones se utilizan para investigaciones biomédicas que conllevan su destrucción o, como mal menor, se donan en adopción a parejas distintas de sus padres biológicos, lo que plantea indudables problemas bioéticos. ${ }^{31}$

También es sabido que, en ocasiones, se utiliza el diagnóstico genético preimplantacional para seleccionar a los mejores embriones para ser implantados y así tratar de hacer las TRA más eficientes. Aunque no podemos profundizar aquí sobre ello, es indudable que el uso del diagnóstico genético preimplantacional va dirigido a seleccionar por razones de salud a los embriones que se consideran de mejor calidad para ser implantados, congelando o desechando a los restantes, lo que indudablemente es una práctica eugenésica.

Pero igualmente plantean las TRA problemas bioéticos relacionados con la donación de gametos, especialmente en lo que hace referencia a si dicha donación debe o no ser anónima, pues ello puede afectar al derecho que los niños nacidos tienen a conocer la identidad de sus padres, y también al derecho de los donantes a salvaguardar su intimidad. Ambas posturas tienen razones objetivas para ser defendidas, pero difícilmente armonizables entre sí. ${ }^{32}$

Otra dificultad bioética que puede darse en torno a las TRA es su utilización para fines sociales ajenos al bien de los hijos e incluso de la dignidad de las mujeres. Especialmente son dos las prácticas más utilizadas: la maternidad subrogada y el «social freezing». La primera afecta directamente tanto a la mujer que gesta a los niños, como al propio bien de éstos, pues con dicha práctica se cosifica a la mujer gestante, utilizándola para fines ajenos a su propio bien, y también al niño producido, el cual, por ser un producto, puede ser sometido a las leyes del mercado. ${ }^{33}$ Estas dificultades bioéticas y morales hacen que la maternidad subrogada esté prohibida en la gran mayoría de los países.

En cuanto al «social freezing», técnica por la que se ofrece a las mujeres congelar sus ovocitos cuando son jóvenes, para utilizarlos más tarde acudiendo a las TRA, y evitando así que queden embara- 
zadas a una edad en la que se presupone que la maternidad puede ser un obstáculo para el desarrollo de sus carreras profesionales. ${ }^{34}$ Naturalmente que esta práctica cosifica igualmente a la mujer, sometiéndola a intereses laborales y económicos ajenos a su propio bien, a la vez que aumenta el riesgo que los embarazos tienen a una edad tardía, y priva a los hijos de tener unos padres jóvenes, con seguridad más aptos para ejercer sus funciones paternas. ${ }^{34}$

Finalmente, las TRA también pueden ser objeto de una comercialización ajena a las más elementales normas deontológicas en la relación médico-paciente, cuando se utiliza «propaganda engañosa» dirigida a la obtención de clientes. Nosotros hemos tratado ampliamente este tema en un artículo anterior, en el que se pone de manifiesto cómo muchas clínicas de reproducción asistida presentan resultados de embarazos y niños nacidos no acordes con su realidad, para mostrar mayor eficacia y así poder incrementar los pingües beneficios económicos de estas clínicas (35).

\section{Limitaciones del estudio}

La principal limitación de este estudio es que, al tratarse de un estudio retrospectivo, puede haber una pérdida de datos no registrados en la historia clínica.

Además, es posible que el registro de ICSI esté infravalorado, porque en muchas madres la FIV se ha llevado a cabo en otro centro distinto al del parto, y no siempre se especifica en las historias el método empleado. Por el mismo motivo, otros datos, como la utilización del diagnóstico genético preimplantatorio y las reducciones fetales, no se han recogido de manera sistemática, al no constar explícitamente en todas las historias.

También se puede considerar una limitación la metodología de inclusión de pacientes, pues, por tratarse de un hospital terciario, se puede dar un aumento de incidencia de patología gestacional y pediátrica, ya que se trata de un centro al que se remiten los casos de 
mayor complejidad y riesgo. No obstante, estas diferencias afectan igualmente a las dos cohortes de pacientes, por lo que no debería alterar la validez interna del estudio.

\section{Conclusión}

En este artículo se objetiva cómo los niños nacidos por TRA, de acuerdo con nuestros propios resultados, así como con los de la bibliografía, presentan más problemas médicos que los nacidos por $\mathrm{CN}$, en especial bajo peso al nacimiento y prematuridad, con las consecuencias clínicas a corto y medio plazo que ello conlleva. También las madres que recurren a estas técnicas presentan patología durante su embarazo en mayor proporción que en las gestaciones habidas por vía natural; sobre todo mayor índice de embarazos múltiples que, como es sabido, suponen mayor riesgo perinatal y obstétrico.

Todos estos problemas médicos hacen que los TRA susciten objetivos dilemas bioéticos, tanto por sí mismas, como por ser utilizados para fines ajenos al propio bien de las madres y de sus hijos, problemas que hemos repasado sucintamente en este trabajo.

\section{Tablas}

Tabla 1. Descripción de las cohortes según edad materna, edad gestacional y peso al nacimiento.

\begin{tabular}{|l|c|c|c|c|}
\hline \multirow{2}{*}{ Variable } & \multicolumn{2}{|c|}{ Grupo CN $(\mathbf{n = 3 0 0})$} & \multicolumn{2}{c|}{ Grupo TRA (n= 300) } \\
\cline { 2 - 5 } & $\begin{array}{c}\text { Media } \\
(\mathrm{SD}) / \mathrm{n}(\%)\end{array}$ & $\begin{array}{c}\text { Mediana } \\
(1 \mathrm{st}, 3 \mathrm{rd} \text { Q. })\end{array}$ & $\begin{array}{c}\text { Media } \\
(\mathrm{SD}) / \mathrm{n}(\%)\end{array}$ & $\begin{array}{c}\text { Mediana } \\
(1 \mathrm{st}, 3 \mathrm{rd} \text { Q. })\end{array}$ \\
\hline Edad materna & $32.62(5.04)$ & $33(29,36)$ & $36.92(4.76)$ & $37(34,40)$ \\
\hline $\begin{array}{l}\text { Edad } \\
\text { gestacional }\end{array}$ & $39.07(2.14)$ & $39.57(38.29,40.43)$ & $37.11(3.37)$ & $37.86(35.57,39.71)$ \\
\hline $\begin{array}{l}\text { Peso } \\
\text { nacimiento }\end{array}$ & $3177.08(581.78)$ & $3200(2900,3572.5)$ & $2686.73(762.33)$ & $\begin{array}{c}2770(2232.5, \\
3271.25)\end{array}$ \\
\hline
\end{tabular}


A. Herrero García, J. Lluna González, J. Aznar Lucea

Tabla 2. Comparación por edad materna.

\begin{tabular}{|c|c|c|}
\hline Edad materna & $\mathbf{C N}(\mathbf{n}=\mathbf{3 0 0})$ & TRA $(\mathbf{n}=\mathbf{3 0 0})$ \\
\hline$<\mathbf{3 5}$ & $185(61.6 \%)$ & $85(28.3 \%)$ \\
\hline $\mathbf{3 5 - 3 9}$ & $92(30.8 \%)$ & $134(44.6 \%)$ \\
\hline $\mathbf{> 4 0}$ & $23(7.6 \%)$ & $81(27 \%)$ \\
\hline
\end{tabular}

Tabla 3. Comparación de tipo de parto, paridad y número de hijos en ambas cohortes.

\begin{tabular}{|c|c|c|c|}
\hline \multirow{2}{*}{ Variable } & Categoría & Grupo CN (n= 300) & Grupo TRA(n= 300) \\
\hline \multirow{3}{*}{ Tipo de parto } & Vaginal & $237(79 \%)$ & $105(35 \%)$ \\
\cline { 2 - 4 } & Cesárea & $63(21 \%)$ & $195(65 \%)$ \\
\hline \multirow{2}{*}{ Paridad } & Único & $289(96.33 \%)$ & $147(49 \%)$ \\
\cline { 2 - 4 } & Múltiple & $11(3.67 \%)$ & $153(51 \%)$ \\
\hline \multirow{3}{*}{ No de hijos } & 1 & $289(96.33 \%)$ & $147(49 \%)$ \\
\cline { 2 - 4 } & 2 & $10(3.33 \%)$ & $148(49.3 \%)$ \\
\cline { 2 - 4 } & 3 & $1(0.33 \%)$ & $5(1.67 \%)$ \\
\hline
\end{tabular}

Tabla 4. Comparación por grupos de edad gestacional.

\begin{tabular}{|c|c|c|}
\hline $\begin{array}{c}\text { Edad gestacional } \\
\text { (semanas) }\end{array}$ & $\mathbf{C N}(\mathbf{n}=\mathbf{3 0 0})$ & TRA $(\mathbf{n}=\mathbf{3 0 0})$ \\
\hline$<\mathbf{2 8}$ & $1(0.33 \%)$ & $12(4 \%)$ \\
\hline $\mathbf{2 8 - 3 2}$ & $7(2.3 \%)$ & $10(3.33 \%)$ \\
\hline $\mathbf{3 2 - 3 6 + 6}$ & $19(7.8 \%)$ & $91(30.3 \%)$ \\
\hline $\mathbf{3 7 - 4 2}$ & $273(91 \%)$ & $187(62.3 \%)$ \\
\hline
\end{tabular}


Tabla 5. Comparación de peso al nacimiento según clasificación acorde con la edad gestacional (EG). PEG (pequeño), AEG (adecuado), GEG (grande).

\begin{tabular}{|c|c|c|}
\hline $\begin{array}{c}\text { Somatometría } \\
\text { al nacimiento }\end{array}$ & $\mathbf{C N}(\mathbf{n}=\mathbf{3 0 0})$ & TRA $(\mathbf{n}=\mathbf{3 0 0})$ \\
\hline PEG & $2(0.66 \%)$ & $19(6.3 \%)$ \\
\hline AEG & $289(96 \%)$ & $278(92.6 \%)$ \\
\hline GEG & $9(3 \%)$ & $3(1 \%)$ \\
\hline
\end{tabular}

Tabla 6. Comparación de los síndromes y cromosomopatías conocidas de ambas cohortes.

\begin{tabular}{|c|c|c|c|c|}
\hline Sexo & Grupo & Síndrome o cromosomopatía & $\begin{array}{c}\text { Retraso } \\
\text { psicomotor }\end{array}$ & Gametos \\
\hline Varón & CN & Síndrome Nance-Horan & Sí & Propios \\
\hline Mujer & TRA & Síndrome de Angelman & Semen \\
Varón & TRA & Deleción cr.1p34.2 & No & Propios \\
\hline Mujer & TRA & $\begin{array}{r}\text { Quimerismo. Cariotipo: chi } 46, \mathrm{XY} \\
{[18] / 46, \mathrm{XX}[17]^{*}}\end{array}$ & No & $\begin{array}{c}\text { Ambos } \\
\text { donantes }\end{array}$ \\
\hline Mujer & TRA & $\begin{array}{c}\text { Sospechaclínica de Síndrome } \\
\text { de Cook, pendiente genética }\end{array}$ & No & Propios \\
\hline Varón & TRA & $\begin{array}{r}\text { Traslocación balanceada. } \\
\text { Cariotipo 46, } \mathrm{XY} t(2: 4)(\mathrm{p} 13, \mathrm{q} 33)\end{array}$ & No & Ovodón \\
\hline Varón & TRA & $\begin{array}{r}\text { Quimerismo. Cariotipo: chi } 46, \mathrm{XY} \\
{[11] / 46, \mathrm{XX}[14]^{*}}\end{array}$ & Sí & Propios \\
\hline Varón & TRA & Delecióncr.22q11.22 & &
\end{tabular}

* [ ] Número de metafases encontradas de cada línea celular. 
Tabla 7. Modelo de regresión logística para analizar la relación entre patología adquirida y TRA, ajustando la edad materna, edad gestacional, tóxicos, malformaciones congénitas y patología gestacional.

\begin{tabular}{|l|c|c|c|c|c|}
\hline \multicolumn{1}{|c|}{ Variables } & $\begin{array}{c}\text { Error } \\
\text { estándar }\end{array}$ & $\begin{array}{c}\text { OR } \\
\text { (Odds Ratio) }\end{array}$ & Inferior.95. & Superior.95. & P valor \\
\hline Edad materna & 0.464 & 1.102 & 0.448 & 2.779 & 0.834 \\
\hline Grupo TRA & 0.2 & 1.926 & 1.301 & 2.857 & 0.001 \\
\hline Tóxicos madres & 0.39 & 1.516 & 0.707 & 3.299 & 0.286 \\
\hline Malf. congénitas & 0.263 & 1.141 & 0.682 & 1.918 & 0.617 \\
\hline $\begin{array}{l}\text { Patología } \\
\text { gestacional }\end{array}$ & 0.205 & 1.328 & 0.889 & 1.989 & 0.167 \\
\hline Edad gestacional & 0.039 & 0.809 & 0.747 & 0.871 & $<0.001$ \\
\hline
\end{tabular}

Gráfico 1. Comparación de la patología adquirida más frecuente.

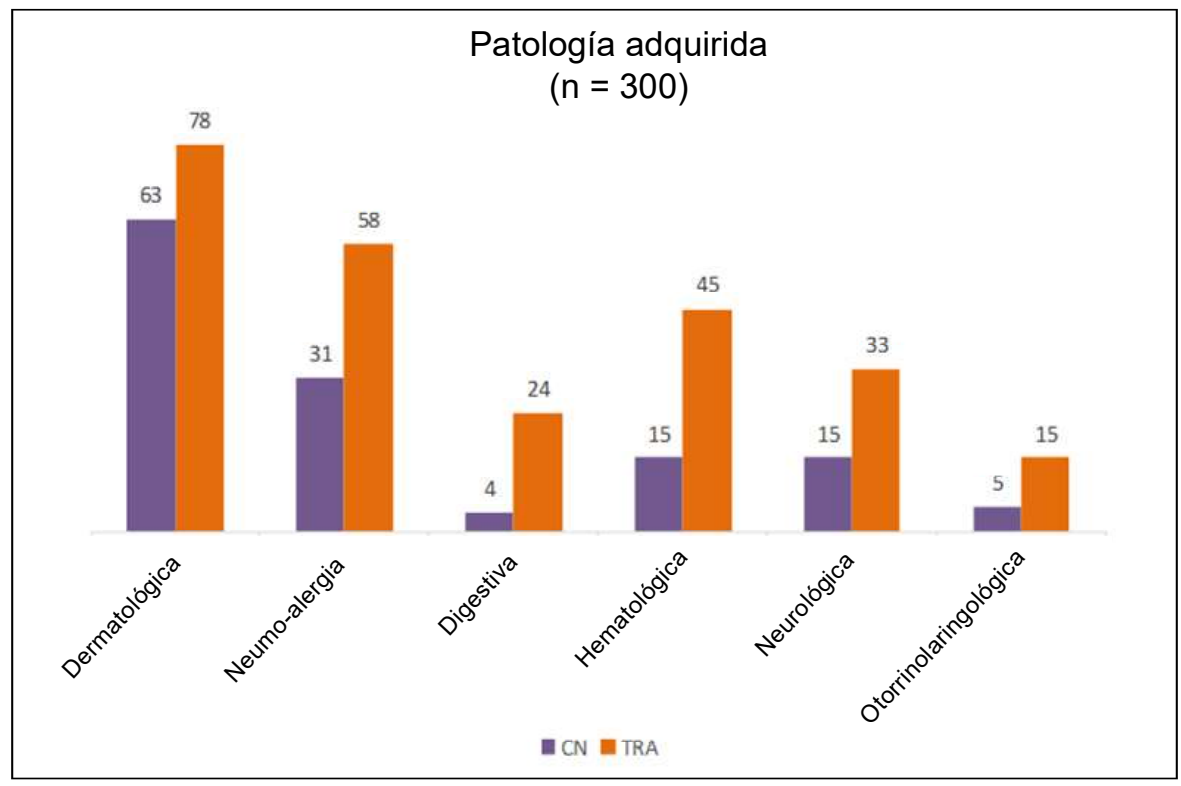


Análisis bioético del impacto de las TRA en la salud de niños y madres

\section{Notas bibliográficas}

1 Nygren, Karl-Gösta; Sullivan, Elizabeth; Zegers-Hochschild, Fernando, MANSOUR, RAGAA, ISHIHARA, OSAMU, ADAMSON, G. DAVID, ET AL. International Committee for Monitoring assisted Reproductive Technology (ICMART) world report: assisted reproductive technology 2003. Fertil Steril. 2011; 95: 2209-22. https:// doi.org/10.1016/j.fertnstert.2011.03.058

2 De Mouzon, Jacques, Goossens, Veerle, Bhattacharya, Samir, Castilla, J, FERRARETTI, ANNA PIA, Korsak, VLADISLAV, ET AL. Assisted reproductive technology in Europe, 2006: results generated from European registers by ESHRE. Hum Reprod. 2010; 25: 1851-62. https://doi.org/10.1093/humrep/des255

${ }^{3}$ FAUSER, BART CJM. Towards the global coverage of a unified registry of IVF outcomes. Reproductive BioMedicine Online. 2019; 38(2): 133-7. https://doi.org/ 10.1016/j.rbmo.2018.12.001

${ }^{4}$ Zheng, Zan, Chen, Letao, Yang, Tubao, Yu, Hong, Wang, Hua, QIn, JIABI. Multiple pregnancies achieved with IVF/ICSI and risk of specific congenital malformations: a meta-analysis of cohort studies. Reprod Biomed Online. 2018; 36(4): 472-482. https://doi.org/10.1016/j.rbmo.2018.01.009

5 Kelley-Quon, lorraine, Tseng, Chi-Hong, Janzen, Carla, Shew, Stephen. Congenital malformations associated with assisted reproductive technology: $A$ California statewide analysis. J Pediatr Surg. 2013; 48(6): 1218-24. https://doi.org/ 10.1016/j.jpedsurg.2013.03.017

${ }^{6}$ Seggers, Jorien, De Walle, Hermien, Bergman, Jorieke, Groen, Henk, HADDERS-AlgRA, MiJnA, Bos, MARLY, ET AL. Congenital anomalies in offspring of subfertile couples: a registry-based study in the northern Netherlands» Fertil Steril. 2015: 103(4): 1001-10. https://doi.org/10.1016/j.fertnstert.2014.12.113

7 Mozafari Kermani, Ramin, Farhangniya, Mansoureh, Shahzadeh, Fazeli, Seyed, Bagheri, Pezhman, Ashrafi, Mahnaz, Vosough Taq Dizaj, Ahmad. Congenital Malformations in Singleton Infants Conceived by Assisted Reproductive Technologies and Singleton Infants by Natural Conception in Tehran, Iran», Int J Fertil Steril. 2018; 11(4): 304-308.

${ }^{8}$ Olson, Christine K, Keppler-Noreuil, Kim, Romitti, Paul A., Budelier, WILLIAM T, RYAN, GINNY, SPARKS, AMY, et al. In vitro fertilization is associated with an increase in major birth defects. Fertil Steril. 2005; 84(5): 1308-15. https:// doi.org/10.1016/j.fertnstert.2005.03.086

9 Giorgione, Veronica, Parazzini, fabio, Fesslova, Vlasta, Cipriani, Sonia, Candiani Massimo, InVERSETti, AnNalisa, Sigismondi, Cristina, Tiberio FranCEScA, CAVORETto, PaOlo IVo. Congenital heart defects in IVF/ICSI pregnancy: systematic review and meta-analysis. Ultrasound Obstet Gynecol. 2018; 51(1): 33-42. https://doi.org/10.1002/uog.18932

${ }^{10}$ Anthony, Sabine, BuitendiJk, Simone E, DorrepaAl Caroline A, Lindner, Katrin, BraAt, Didi D, Den Ouden, ANne Mieke. Congenital malformations in 4224 
children conceived after IVF. Hum Reprod. 2002; 17(8): 2089-95. https://doi.org/ 10.1093/humrep/17.8.2089

${ }^{11}$ Halliday, Jane, LeWis, Sharon, Kennedy, JoAnne, Burgner, DaVid P, JuONAla, MARKUS, HAMmARBERG, KARIN, ET AL. Health of adults aged 22 to 35 years conceived by assisted reproductive technology. Fertil Steril. 2019; 112(1): 130-139. https://doi.org/10.1016/j.fertnstert.2019.03.001

${ }^{12}$ Bonduelle, Maryse, Liebaers, Inge, Deketelaere, Veerle, Derde, MariePaule, Camus, M, Devroey, Paul, Van Steirteghem, André. Neonatal data on a cohort of 2889 infants born after ICSI (1991-1999) and of 2995 infants born after IVF (1983-1999). Hum Reprod. 2002; 17(3): 671-94. https://doi.org/10.1093/ humrep/17.3.671

${ }^{13}$ Wen, Juan, Jiang, Jie, Ding, Chenyue, Dal, Juncheng, Liu, Yao, Xia, Yankal, et $A L$. Birth defects in children conceived by in vitro fertilization and intracytoplasmic sperm injection: a meta-analysis. Fertil Steril. 2012; 97(6): 1331-7. https://doi.org/ 10.1016/j.fertnstert.2012.02.053

${ }^{14}$ Hoorsan, Hayeden, Mirmiran, Parvin, Chaichian, Shahla, Moradi, Yousef, HoORSAN, ROZA, JESMI, FATEMEH. Congenital Malformations in Infants of Mothers Undergoing Assisted Reproductive Technologies: A Systematic Review and Metaanalysis Study. J Prev Med Public Health. 2017; 50(6): 347-60. https://doi.org/ 10.3961/jpmph.16.122

15 Davies, Michael J, Moore, Vm, Willson, KJ, Van Essen, P, Priest, K, Scott, H., HAAN, EA, CHAN, A. Reproductive technologies and the risk of birth defects. N. Engl J Med. 2012; 366,19: 1803-13. https://doi.org/10.1056/nejmoa1008095

${ }^{16}$ SAGOT, PAUL, BECHOUA, SHALIHA, FERDYNUS, CYRIL, FACY, AUDREY, FLAMM, XENIA, GOUYON JEAN BERNARD, JIMENEZ, CLÉMENT. Similarly increased congenital anomaly rates after intrauterine insemination and IVF technologies: a retrospective cohort study. Hum Reprod. 2012; 27(3): 902-9. https://doi.org/10.1093/humrep/der443

17 HVidTJøRn, Dorte, Schieve, Laura, Schendel, DiAnA, JacoBsson, Bo, Svaerke, Claus, Thorsen, Poul. Cerebral palsy, autism spectrum disorders, and developmental delay in children born after assisted conception: a systematic review and meta-analysis. Arch Pediatr Adolesc Med. 2009; 163(1): 72-83. https:// doi.org/10.1001/archpediatrics.2008.507

18 Sandin, Sven, NygRen, Karl-Gösta, Iliadou, Anastasia, Hultman Christina M, REICHENBERG, ABRAHAM. Autism and mental retardation among offspring born after in vitro fertilization. JAMA. 2013; 310(1): 75-84. https://doi.org/10.1001/jama. 2013.7222

${ }^{19}$ Källén, Bengt, Finnström, Orvar, Nygren, Karl-Gösta, Otterblad OlausSON, PETRA. Asthma in Swedish children conceived by in vitro fertilization. Arch Dis Child. 2013; 98(2): 92-6. https://doi.org/10.1136/archdischild-2012-301822

20 Klemetti, Reija, Sevón, Tilna, Gissler, Mika, Hemminki, Elina. Health of children born after ovulation induction. Fertil Steril. 2010; 93(4): 1157-68. https:// doi.org/10.1016/j.fertnstert.2008.12.025 
${ }^{21}$ Meister, Théo A, Rimoldi, Stefano F, Soria, Rodrigo, Von ArX, Robert, MesSERLI, Franz H, SARTORI, Claudio, ScherRer, URS, ReXHAJ, EMrush. Association of assisted reproductive technologies with arterial hypertension during adolescence. J Am Coll Cardiol. 2018; 72(11): 1267-1274. https://doi.org/10.1016/j.jacc. 2018.06.060

${ }^{22}$ Belva, Florence, Bonduelle, Maryse, Roelants, Mathieu, Michielsen, Dolf, Van Steirteghem, André, Verheyen Greta, Tournaye, Herman. Semen quality of young adult ICSI offspring: the first results. Hum Reprod. 2016; 31(12): 28112820. https://doi.org/10.1093/humrep/dew245

${ }^{23}$ Berntsen, Sine, Söderström-Anttila, Viveca, Wennerholm, Ulla Britt, Laivuori, hannele, loft, Anne, Oldereid, Nan B, Romundstad, Liv Bente, BERgh, ChristinA, PINBORg, ANJA. The health of children conceived by ART: «The chicken or the egg?» Hum Reprod Update. 2019; 25(2): 137-158. https://doi.org/ 10.1093/humupd/dmz001

${ }^{24}$ Cabañas, Fernando, lópez-Azorín, Manuela, Pellicer, Antonio. Assisted reproduction techniques and the health of the newborn. An Pediatr. 2009; 70(4): 319-22.

25 JiAng, ZiRU, Wang, YinYu, LiN, JING, XU, JingJing, Ding, Guolian, Huang, HEFENG. Genetic and epigenetic risks of assisted reproduction. Best Pract Res Clin Obstet Gynaecol. 2017; 44: 90-104. https://doi.org/10.1016/j.bpobgyn. 2017.07.004

${ }^{26}$ KÄLLÉN, BENGT. Maternal morbidity and mortality in in-vitro fertilization. Best Pract Res Clin Obstet Gynaecol. 2008; 22(3): 549-58.

27 Instituto Nacional DE Estadística. Patrones de mortalidad en España. 2016. Disponible en: https://www.mscbs.gob.es/estadEstudios/estadisticas/estadisticas/ estMinisterio/mortalidad/docs/Patrones_Mortalidad_2016.pdf

${ }^{28}$ Ley Orgánica 9/1985, de 5 de julio, de reforma del artículo 417 bis del Código Penal. BOE Número: 166, 12/07/1985 [Disposición derogada], p. 22041.

${ }^{29}$ Ley Orgánica $2 / 2010$, de 3 de marzo, de salud sexual y reproductiva y de la interrupción voluntaria del embarazo. BOE Número: 55, 04/03/2010, Disposición $n^{\circ} 3514$, p. 21001-21014.

30 AzNAR, Justo, Mínguez, José ÁNGEL. Loss of human embryos secondary to in vitro fertilization. Medicina e Morale. 2012; 4: 613-6.

${ }^{31}$ Aznar, Justo, Martínez, Miriam, NavarRo, Pedro. Moral assessment of frozen human embryo adoption in the light of the Magisterium of the Catholic Church», Acta Bioeth. 2017; 23: 137-49. https://doi.org/10.4067/s1726-569x2017000 100137

${ }^{32}$ AZNAR, JUSTO, TUDELA, JULIO. Ethical aspects of assisted reproduction techniques. In: Innovations. En: Assisted Reproduction Technology. Intech Open, London; 2020 (in press).

${ }^{33}$ Aznar, Justo, Martínez Peris, Miriam. Gestational Surrogacy: Current View. Linacre Q. 2019; 86: 56-7. 
A. Herrero García, J. Lluna González, J. Aznar Lucea

${ }^{34}$ AzNAR, JUSto, TUdelA, JULIO. Social freezing: analysis of an ethical dilemma. Ethics Med. 2019; 35: 161-70.

${ }^{35}$ AZnAR, JuAN, TUdela, JULIO, AZnAR Justo. Analysis of the truth in advertising on the efficacy provided by assisted reproduction clinics. Acta Bioeth. 2017; 23: 311-325. https://doi.org/10.4067/s1726-569x2017000200311 